\title{
Measurement of Milwaukee Brace Pad Pressure in Adolescent Round Back Deformity Treatment
}

\author{
Taher Babaee ${ }^{1}$, Mojtaba Kamyab ${ }^{1}$, Amir Ahmadi ${ }^{2}$, Mohammad Ali Sanjari ${ }^{3}$, Mohammad Saleh Ganjavian ${ }^{4}$ \\ ${ }^{1}$ Department of Orthotics and Prosthetics, School of Rehabilitation Sciences, Iran University of Medical sciences, Tehran, Iran \\ ${ }^{2}$ Department of Physiotherapy, School of Rehabilitation Sciences, Iran University of Medical sciences, Tehran, Iran \\ ${ }^{3}$ Department of Rehabilitation Basic Sciences, School of Rehabilitation, and Biomechanics Lab, \\ Rehabilitation Research Center, Iran University of Medical sciences, Tehran, Iran \\ ${ }^{4}$ Department of Orthopaedic Surgery, Shafa Yahyaiian hospital, Iran University of Medical Sciences, Tehran, Iran
}

Study Design: In this prospective study, we measured the pad pressures of the Milwaukee brace in adolescent hyperkyphosis treatment. Purpose: We evaluated the skin-brace interface forces exerted by the main pads of the Milwaukee brace.

Overview of Literature: A fundamental factor associated with brace effectiveness in spinal deformity is pad force adjustment. However, few studies have evaluated the in-brace force magnitude and its effect on curve correction.

Methods: Interface forces at four pads of the Milwaukee brace were measured in 73 patients withround back deformity (mean age, $14.04 \pm 1.97$ years [range, 10-18]; mean initial Cobb angle, $67.70^{\circ} \pm 9.23^{\circ}$ [range, $50^{\circ}-86^{\circ}$ ]). We used a modified aneroid sphygmomanometer to measure the shoulder and kyphosis pad pressures. Each patient underwent measurement in the standing and sitting positions during inhalation/exhalation.

Results: The mean pad pressures were significantly higher in the standing than in thesitting position, and significantly higher pressures were observed during inhalation compared toexhalation $(p=0.001)$.There were no statistically significant differences between right and left shoulder pad pressures ( $p>0.05)$; however, the pressure differences between the right and left kyphosis pads were statistically significant $(p<0.05)$. In a comparison of corrective forces with bracing for less or more than 6 months, corrective force was larger with bracing for less than 6 months $(p=0.02)$. In the standing position, there were no statistically significant correlations between pad pressures and kyphosis curve correction.

Conclusions: In the sitting position, there was a trend toward lower forces at the skin-brace interface; therefore, brace adjustment in the standing position may be useful and more effective. There was no significant correlation between the magnitude of the pad pressures and the degree of in-brace curve correction.

Keywords: Pressure; Brace; Adolescent kyphosis; Sphygmomanometer; Spinal deformity

\section{Introduction}

The Scoliosis Research Society has reported the normal range of thoracic kyphosis as $20^{\circ}$ to $45^{\circ}$ in growing ado- lescents [1]. Generally, a kyphotic curve greater than $45^{\circ}$ in the thoracic spine is known as hyperkyphosis and requires active treatment, such as bracing and exercise, to control curve progression [2]. Hyperkyphosis is caused by

\footnotetext{
Received Nov 26, 2016; Revised Jan 13, 2017; Accepted Feb 5, 2017

Corresponding author: Mojtaba Kamyab

Department of Orthotics and Prosthetics, School of Rehabilitation Sciences, Iran University of Medical sciences,

Madadkaran Avenue, Shahnazari St., Madar square, Mirdamad Blvd., Tehran, Iran

Tel: +98-21-2222-0947 (Ext 255), Fax: +98-21-2222-0946, E-mail: kamyab.m@iums.ac.ir
} 
spine fractures, neuromuscular diseases, Scheuermann's disease, and unknown mechanisms. One of the common causes is Scheuermann's disease, with an incidence of $0.4 \%$ to $10 \%$ [3], which affects adolescents aged 10 to 16 years [4]. These patients have a thoracic round back deformity, often with accompanying compensatory increased lumbar lordosis. The position of the head is often in a forward posture and the shoulders are positioned internally rotated.

Bracing is the most effective nonoperative treatment for patients with flexible kyphotic deformity, and its effectiveness has been proven in various studies [5,6]. In accordance with the three-point pressure principle, the brace provides adequate corrective force at the apex of the curve and reduction of lumbar lordosis, which are essential in hyperkyphosis treatment. For patients with a progressive curve at a thoracic apex of T8 or above, the Milwaukee brace is the best choice for application of a three-point corrective force at the mid-thoracic level [7]. For use in extending the trunk, the Milwaukee brace consists of two left-right pairs of corrective pads (kyphosis pads), which apply force anteriorly directed at and just inferior to the apex of the curve, and shoulder pads, which apply force posteriorly directed through shoulder outriggers [8].

Adjustment of pad force is a fundamental issue associated with in-brace curve correction and brace effectiveness, but few studies have evaluated the magnitude of these corrective forces [9-11]. For example, Loukos et al. [9] assessed interface forces in a dynamic derotation brace by a PEDAR system in different postures. Pham et al. [11] evaluated the pressure of corrective pads in the Chêneau brace by the Tekscan Clin Seat type 5315 sensor. As different braces and measurement tools were used in these studies, consistent results were not achieved. On the other hand, pressure sensors used in previous studies are not always available in clinical settings, and are expensive. A modified sphygmomanometer is a reliable pressure measurement tool that is available in every clinic for use in pad pressure measurement [12]. Few studies have evaluated the corrective forces acting upon the body during brace treatment of spinal deformities, and adjustment of the pad pressure is still usually empirical.

To the best of our knowledge, no study has measured pad pressures in adolescent round back deformity treated with the Milwaukee brace. Thus, we aimed to analyze the pressure exerted by each pad of the Milwaukee brace using a modified sphygmomanometer, investigating the variation in these pressures with body posture and assessing the relationship between mean pressure value and degree of in-brace curve correction in adolescents with round back deformity.

\section{Materials and Methods}

\section{Participants}

This cross-sectional multicenter study was performed on 73 patients ( 45 girls, 28 boys) who attended spine clinics in Iran for a routine brace check and adjustment. The study was approved by the Human Research Ethics Committee of Tehran, Iran University of Medical Sciences (Letter no. 1215). The procedures were explained to the volunteers and the participants completed the consent forms. All participants who met the inclusion criteria had round back deformity, were aged 10 to 18 years, and were treated with the Milwaukee brace, based on diagnosis by an experienced spine surgeon. All patients with some flexibility across the kyphotic curve were candidates for brace treatment [5]. Patient mean age was $14.04 \pm 1.97$ years (range, 10-18 years). The mean initial Cobb angle at the time of brace prescription was $67.70^{\circ} \pm 9.23^{\circ}$ (range, $50^{\circ}$ $86^{\circ}$ ). The in-brace Cobb angle was $46.30^{\circ} \pm 10.9^{\circ}$ (range, $25^{\circ}-70^{\circ}$ ). For patients with a Cobb angle greater than $74^{\circ}$, surgery was proposed but refused by parents. Therefore, the physician proposed Milwaukee brace treatment for them.

\section{Procedure}

The kyphosis and shoulder pads of the Milwaukee brace were made of thermoplastic material ( $4 \mathrm{~mm}$ thick). A metal wire marker was used around the pads to control their appropriate position at the time of X-ray measurement [13]. Pad surfaces in contact with the body were covered by a layer of soft material (Plastazote, $5 \mathrm{~mm}$ thick). Kyphosis pads were attached on posterior uprights within $3 \mathrm{~cm}$ from their inner edge to follow the coronal diameter of the vertebrae (Fig. 1). To increase the amount of corrective force in the Milwaukee brace, the orthotist adjusted the posterior paraspinal bars in an antikyphotic posture; the shoulder outriggers were formed to exert more posterior-directed force on the deltopectoral muscles. All measurements were performed after the brace was adjusted. Two modified aneroid sphygmomanometers 
(Exacta-Riester 0124, Rudolf Riester GmbH, Jungingen, Germany) were used to measure pad pressures. Pressure measurements were performed for the shoulder pads with a neonatal blood pressure cuff $\left(4 \times 6 \mathrm{~cm}^{2}\right)$ and for kyphosis pads with a pediatric cuff $\left(13 \times 8 \mathrm{~cm}^{2}\right)$. The sphygmomanometers were modified by removing the outer shell of

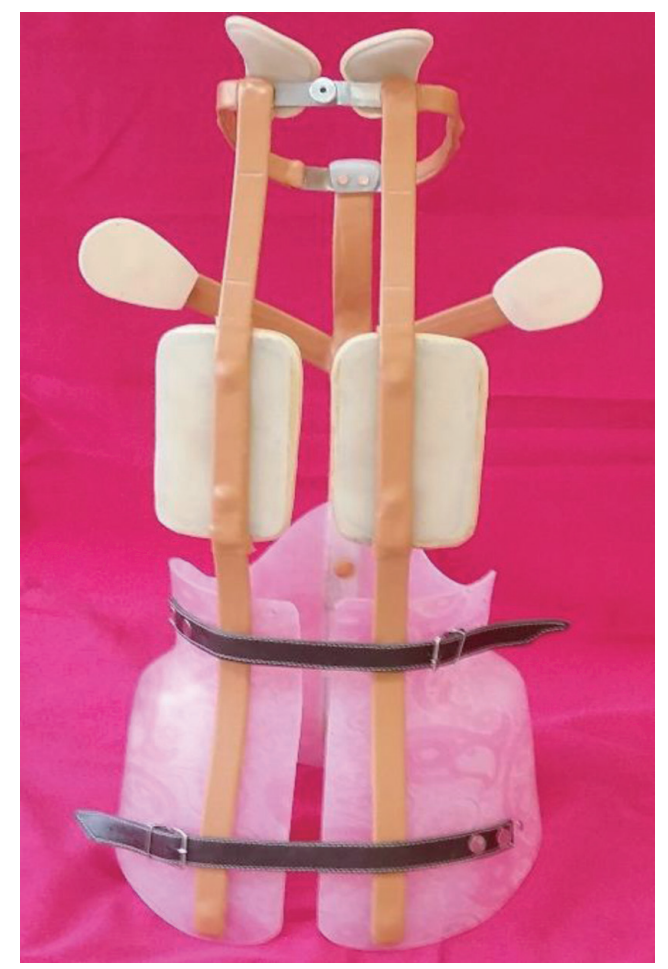

Fig. 1. The Milwaukee brace for hyperkyphosis. the pressure cuff for better operation. For calibration of the sphygmomanometer, at each trial, after the cuff was inflated to the desired level of $20 \mathrm{~mm} \mathrm{Hg}$, the examiner pressed and released the cuff 3 to 4 times. The inflation was readjusted until constant readings were obtained after repeated presses [14]. To remove wrinkles, the cuffs of the modified sphygmomanometers were filled with air to 1-cm thickness.

Measurements were performed with the patient in the sitting and standing positions during inhalation and exhalation of tidal breathing.In the standing position, cuffs were placed longitudinally between the pads and the patient's body (Fig. 2). The examiners asked patients to put their hands at their sides and retract their necks. Neck retraction is one component of correcting forward head posture in hyperkyphosis [15]. The numbers on the pressure gauge dial moved up/down with inhalation/exhalation and were recorded at the fifth breath. Measurement was repeated if any perturbation, such as deep breathing, affected the test. In the sitting position, the participants were asked to sit on an adjustable chair with their knees flexed to $90^{\circ}$ and their feet in contact with the ground [16]. The patient's arms were at their sides with the hands on the thighs and the neck in a retracted position. They were asked to maintain their posture during the test. Measurements of shoulder pad pressures were conducted first in the sitting and standing positions. For each patient, a total of 16 measurements were performed in the sitting and
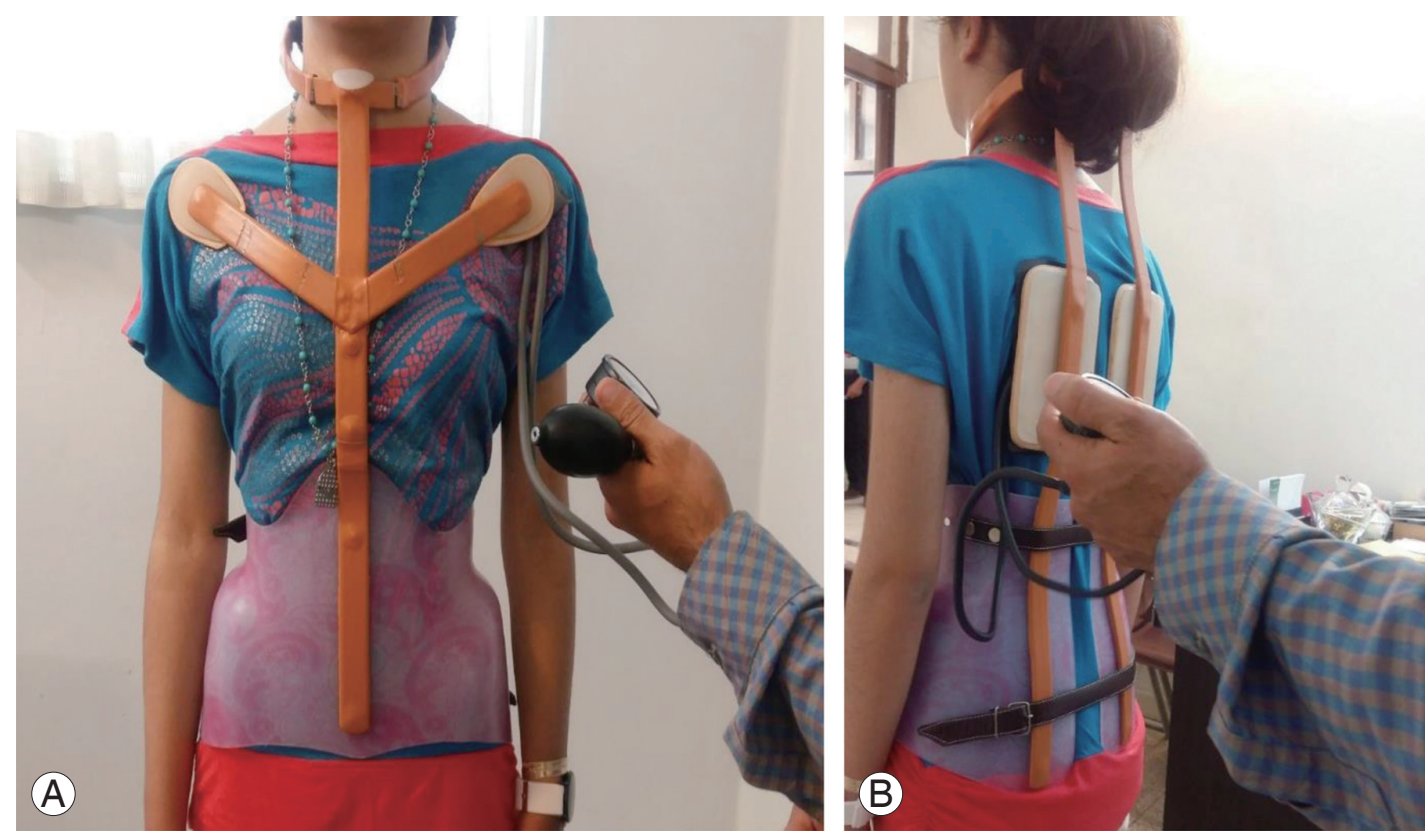

Fig. 2. (A, B) Two sides of the Milwaukee brace and the pressure cuff placement. 
standing positions during inhalation/exhalation with tidal breathing. It is generally believed that the corrective force is greater in the new braces. Therefore, to evaluate corrective forces exerted by the brace between patients who had been in braces for less than and more than 6 months, the participants were categorized in two groups. The mean inbrace curve correction was measured according to comparison of the pretreatment and the last in-brace lateral radiographs. To asses in-brace force, the average of pressure values for four pads of the Milwaukee brace in the standing position were computed.

\section{Statistical analysis}

Statistical analysis software ver. 17 (SPSS, Inc., Chicago, IL, USA) was used for calculations. Using the $t$-test for paired samples, we compared mean pad pressures in a standing and a sitting position. The independent sample $t$-test was used to evaluate the differences between the average scores for the right and left shoulder pads and right and left kyphosis pads in the standing position. Pearson's correlation coefficient was used to analyze the relationship between the mean pressure value in the standing position and degree of kyphosis curve correction. The significance level was set to 0.05 for all tests.

\section{Results}

Table 1 shows the mean pressure values for the four pads of the Milwaukee brace in the standing and sitting positions during tidal breathing. Pad pressures were significantly higher in the standing than in the sitting position as well as during inhalation compared to exhalation $(p<0.05)$. There were no significant differences in average scores (average between inhalation and exhalation) between the right and left shoulder pad pressures ( $p>0.05)$ (Table 2). However, right kyphosis pads exerted greater pressure than left pads $(p<0.05)$. The mean pad pressures were significantly different between patients who underwent bracing for less or more than 6 months from the beginning of treatment $(p=0.02)$. In the standing position, no significant correlations were observed between pad pressures and kyphosis curve correction $(r=0.89, p=0.4)$. The mean brace wearing duration for the 73 participants was 10.98 months (range, 3-36 months), and the mean curve correction was $31.61 \%$ for all participants. The wearing time was obtained from the brace delivery records.

As shown in Table 3 , there was a statistically significant difference in brace force between patients with less than and more than 6 months of bracing.

Table 1. Means \pm standard deviations and paired sample $t$-test comparison measurement results for shoulder/kyphosis pads pressure in standing and sitting position during tidal breathing

\begin{tabular}{|c|c|c|c|c|c|c|}
\hline \multirow{2}{*}{$\begin{array}{l}\text { Mean pad pressure } \\
(\mathrm{mm} \mathrm{Hg})\end{array}$} & \multicolumn{3}{|c|}{ Standing position } & \multicolumn{3}{|c|}{ Sitting position } \\
\hline & Inhalation & Exhalation & $p$-value & Inhalation & Exhalation & $p$-value \\
\hline Right shoulder & $68.99 \pm 21.95$ & $62.66 \pm 21.41$ & 0.001 & $62.66 \pm 20.75$ & $56.48 \pm 20.20$ & 0.001 \\
\hline Left shoulder & $68.10 \pm 23.84$ & $61.28 \pm 23.14$ & 0.001 & $61.79 \pm 23.54$ & $55.91 \pm 22.69$ & 0.001 \\
\hline Right kyphosis & $53.73 \pm 14.86$ & $46.87 \pm 13.85$ & 0.001 & $43.81 \pm 14.57$ & $37.34 \pm 12.33$ & 0.001 \\
\hline Left kyphosis & $48.19 \pm 13.08$ & $41.46 \pm 11.82$ & 0.001 & $40.00 \pm 13.07$ & $33.43 \pm 10.88$ & 0.001 \\
\hline
\end{tabular}

Table 2. Means \pm standard deviations and independent sample $t$-test comparison for average scores between the right/left shoulder and right/left kyphosis pads in standing position

\begin{tabular}{lccc} 
Pads & Right side $(\mathrm{mm} \mathrm{Hg})$ & Left side $(\mathrm{mm} \mathrm{Hg})$ & $p$-value \\
Shoulder & $65.82 \pm 21.61$ & $64.95 \pm 23.58$ & 0.82 \\
Kyphosis & $50.30 \pm 14.14$ & $44.83 \pm 12.32$ & 0.01 \\
\hline
\end{tabular}

Table 3. Mean pressure value based on brace wearing time per month

\begin{tabular}{lccc} 
Brace wearing time & Lower than $6 \mathrm{mo}(\mathrm{n}=28)$ & Higher than $6 \mathrm{mo}(\mathrm{n}=45)$ & $p$-value \\
Average in-brace force $(\mathrm{mm} \mathrm{Hg})$ & $61.41 \pm 15.06$ & $53.35 \pm 13.76$ & 0.02 \\
\hline
\end{tabular}




\section{Discussion}

Result of the current study showed that the mean resultant pressure exerted on the patient's body was higher during inhalation than during exhalation. Also, in-brace pressure in the standing position was higher than that in the sitting position. In contrast, van den Hout et al. [16] and Loukos et al. [9] reported higher pad pressures in the sitting than in the standing position, using a Boston brace and dynamic derotation brace. However, Pham et al. [11] and Aubin et al. [17] did not observe any differences in thoracic pad pressures of the thoracolumbosacral orthosis in either the sitting or standing position in scoliotic patients. One likely explanation is that contact of the Milwaukee brace neck ring with the patient's neck invokes an uncomfortable, posteriorly directed stimulus superior to the apex of the curve. The mechanism of the Milwaukee brace to reduce the thoracic hyperkyphosis relies on decreasing the lumbar lordosis, applying a passive force at the mid thoracic level, which exerts an anteriorly directed force at and just below the apex of the curve, and active response of the patient to the shoulder outriggers and neck ring. This reaction is known as a kinesthetic reminder, and is one of the kyphosis curve correction components that are performed actively by the patients. Another explanation could be that flexion of the hips and knees in the sitting position places the ilium in backward rotation and decreases lumbar lordosis [18]. The reduction in lumbar lordosis in the brace is a "strategic orthotic maneuver" that can decrease thoracic kyphosis, and consequently, pad pressure [3]. Therefore, adjustment of the Milwaukee brace pad pressures in the standing position appears to be a better approach.

Some studies were conducted to analyze the skinbrace interface pressure at different strap tensions while the subjects take on different positions and activities $[9,11,17,19,20]$. In these studies, results showed that tightening the brace's straps can produce a higher in-brace force, but different brace types, various curve patterns, and dissimilar application methods were used; the resulting data disparity hindered any conclusion. In a study by Pham et al. [11], strap tension was increased by $2 \mathrm{~cm}$ and the mean in-brace pressures were increased significantly. In another study by Loukos et al. [9], there was a significant increase in the in-brace pressure when the strap tension was maximum. Indeed, if brace effectiveness depends on how tightly the brace should be fastened, a standard tension may be set in each strap. In the current study, before beginning the procedure, the orthotist fastens the brace on the patient's body with a $1 \mathrm{~cm}$ distance between the two posterior borders of the pelvic section. This distance was recommended by patients treating physicians.

From the biomechanical perspective, applying the equivalent pressure across the main pads of the brace upon the body is one of the key components of bracing effectiveness, which is in agreement with the three-point pressure principle of brace treatment [10]. The findings in the current study showed that the magnitudes of pressures at the right and left shoulder pads were similar; however, the right and left kyphosis pads had different pressures. This may be because patients are more sensitive to pressure at the glenohumeral joint region due to less overlying soft tissue and greater sensory feedback. In the thoracic region, the kyphosis pad forces are applied over a wider area, and patients do not perceive significant pressure differences. Therefore, the responsiveness of patient feedback for applied force in the shoulder region is higher than that for the thoracic spine region. Moreover, the pressure of the right kyphosis pads was greater than those on the left, which may reflect the handedness of the orthotists, all of whom were right-handed. However, this premise was not evaluated statistically, and thus, further research is needed.

In this study, the results of analysis of in-brace force based on duration of wear showed that the pads exerted more force in patients who had been wearing braces for less than 6 months than in those who had been wearing braces for more than 6 months $(p<0.02)$. The reason for greater pressure in the first months after brace wearing could be that most viscoelastic changes and curve correction occur in the initial bracing period [21,22]. van den Hout et al. [16] reported that new braces ( $<6$ months) had slightly more corrective force than old braces ( $>6$ months), although the difference was not statistically significant.

Based on the literature, one of the important predictive factors for the outcome of orthotic treatment in spinal deformities is the degree of initial in-brace curve correction $[22,23]$. In the current study, the mean in-brace curve correction was $21.63^{\circ}(31.6 \%)$, achieved after an average of 10.9 months. However, Bradford et al. [24] found that the mean in-brace curve correction was $30^{\circ}(40.7 \%)$ after an average period of 8.9 months. The difference may be attributed to the initial Cobb angle; in Bradford's study, the mean initial Cobb angle before initiation of brace treatment was $58.9^{\circ}$, but in the current study, the initial Cobb angle was $67.8^{\circ}$. 
It is generally believed that an increase in corrective force leads to a higher in-brace curve correction and a better control of the spinal deformity. Results of the current study showed that, in the standing position, the overall mean pressure at the four pads of the Milwaukee brace was $56.47 \pm 20.57 \mathrm{~mm} \mathrm{Hg}$ for a Cobb angle correction of $31.61 \%$. We found no significant correlation between the magnitude of pad pressures and the degree of achieved radiographic in-brace curve correction $(r=-0.08, p=0.47)$. These findings were confirmed by previous studies for adolescents with idiopathic scoliosis who had been managed with the thoracolumbosacral orthoses $[9,11,16]$.

The magnitude of in-brace force or pressure alone is not enough to achieve optimal curve correction [23]. Many other parameters can affect the outcome of bracing in adolescents with spinal deformities. These parameters include sex of the patient and orthotist, joint flexibility, and exercise while wearing the brace. Therefore, it is suggested that the mentioned parameters be considered in the future studies. To the best of our best knowledge, there is no study available for analyzing the magnitude of in-brace corrective force for adolescents with hyperkyphosis. However, one limitation of the current study is that we only measured the pad pressures of the Milwaukee brace in the sitting and standing positions. Also, the external validity of this study was limited to adolescents with round back deformity. Therefore, investigating in-brace pressure for other spinal deformities, such as scoliosis, in different everyday tasks and postures is recommended for future studies.

\section{Conclusions}

This study provides data about the magnitude of interface corrective pressures of the Milwaukee brace in adolescents with round back deformity. The use of a modified sphygmomanometer for pressure measurement at each visit for a routine brace check can be a useful way to optimize fit and effectiveness. As there is a trend toward higher pressures in the standing position, adjustment of the Milwaukee brace in the standing position appears to be a useful approach.

\section{Conflict of Interest}

No potential conflict of interest relevant to this article was reported.

\section{Acknowledgments}

This study was supported by the research committee of Iran University of Medical Sciences.

\section{References}

1. Fon GT, Pitt MJ, Thies AC Jr. Thoracic kyphosis: range in normal subjects. AJR Am J Roentgenol 1980;134:979-83.

2. Bradford DS. Vertebral osteochondrosis (Scheuermann's kyphosis). Clin Orthop Relat Res 1981;(158):83-90.

3. John D, Hsu JW, Fisk JR. Orthoses for spinal deformities. In: Hsu JD, Michael JW, Fisk JR; American Academy of Orthopaedic Surgeons, editors. AAOS atlas of orthoses and assistive devices. 4th ed. Philadelphia: Elsevier; 2008. p.125-39.

4. Bezalel T, Kalichman L. Improvement of clinical and radiographical presentation of Scheuermann disease after Schroth therapy treatment. J Bodyw Mov Ther 2015;19:232-7.

5. Hart ES, Merlin G, Harisiades J, Grottkau BE. Scheuermann's thoracic kyphosis in the adolescent patient. Orthop Nurs 2010;29:365-71.

6. Montgomery SP, Erwin WE. Scheuermann's kyphosis: long-term results of Milwaukee braces treatment. Spine (Phila Pa 1976) 1981;6:5-8.

7. Papagelopoulos PJ, Mavrogenis AF, Savvidou OD, Mitsiokapa EA, Themistocleous GS, Soucacos PN. Current concepts in Scheuermann's kyphosis. Orthopedics 2008;31:52-8.

8. McIntosh AL, Sucato DJ. Scheuermann's kyphosis. Curr Opin Orthop 2007;18:536-43.

9. Loukos I, Zachariou C, Nicolopoulos C, Korres D, Efstathopoulos N. Analysis of the corrective forces exerted by a dynamic derotation brace (DDB). Prosthet Orthot Int 2011;35:365-72.

10. Mac-Thiong JM, Petit Y, Aubin CE, Delorme S, Dansereau J, Labelle $\mathrm{H}$. Biomechanical evaluation of the Boston brace system for the treatment of adolescent idiopathic scoliosis: relationship between strap tension and brace interface forces. Spine (Phila Pa 1976) 2004;29:26-32.

11. Pham VM, Houilliez A, Schill A, Carpentier A, Herbaux B, Thevenon A. Study of the pressures applied by a Cheneau brace for correction of adolescent 
idiopathic scoliosis. Prosthet Orthot Int 2008;32:34555.

12. Kaegi C, Thibault MC, Giroux F, Bourbonnais D. The interrater reliability of force measurements using a modified sphygmomanometer in elderly subjects. Phys Ther 1998;78:1095-103.

13. Ganjavian MS, Behtash H, Ameri E, Khakinahad M. Results of Milwaukee and Boston braces with or without metal marker around pads in patients with idiopathic scoliosis. Acta Med Iran 2011;49:598-605.

14. Lusardi MM, Bohannon RW. Hand grip strength: comparability of measurements obtained with a jamar dynamometer and a modified sphygmomanometer. J Hand Ther 1991;4:117-22.

15. Wenger DR, Frick SL. Scheuermann kyphosis. Spine (Phila Pa 1976) 1999;24:2630-9.

16. van den Hout JA, van Rhijn LW, van den Munckhof RJ, van Ooy A. Interface corrective force measurements in Boston brace treatment. Eur Spine J 2002; 11:332-5.

17. Aubin CE, Labelle H, Ruszkowski A, et al. Variability of strap tension in brace treatment for adolescent idiopathic scoliosis. Spine (Phila Pa 1976) 1999;24:34954.

18. Cho IY, Park SY, Park JH, Kim TK, Jung TW, Lee
HM. The effect of standing and different sitting positions on lumbar lordosis: radiographic study of 30 healthy volunteers. Asian Spine J 2015;9:762-9.

19. Wong MS, Evans JH. Biomechanical evaluation of the Milwaukee brace. Prosthet Orthot Int 1998;22:54-67.

20. Chase AP, Bader DL, Houghton GR. The biomechanical effectiveness of the Boston brace in the management of adolescent idiopathic scoliosis. Spine (Phila Pa 1976) 1989;14:636-42.

21. Castro FP Jr. Adolescent idiopathic scoliosis, bracing, and the Hueter-Volkmann principle. Spine J 2003;3: 180-5.

22. Chan SL, Cheung KM, Luk KD, Wong KW, Wong MS. A correlation study between in-brace correction, compliance to spinal orthosis and health-related quality of life of patients with Adolescent Idiopathic Scoliosis. Scoliosis 2014;9:1.

23. Katz DE, Durrani AA. Factors that influence outcome in bracing large curves in patients with adolescent idiopathic scoliosis. Spine (Phila Pa 1976) 2001; 26:2354-61.

24. Bradford DS, Moe JH, Montalvo FJ, Winter RB. Scheuermann's kyphosis and roundback deformity. Results of Milwaukee brace treatment. J Bone Joint Surg Am 1974;56:740-58. 